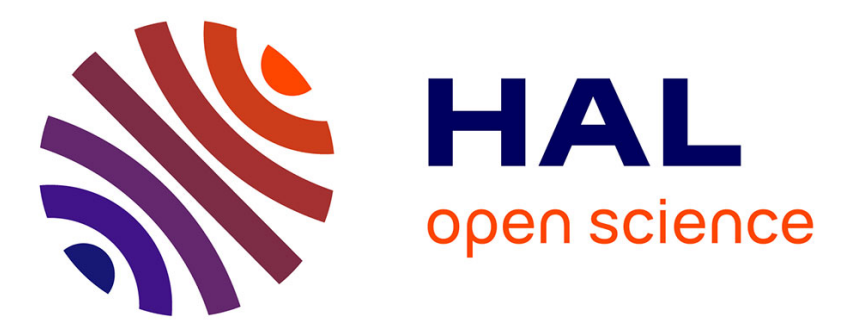

\title{
Two temperature model for thermoacoustic sound generation in thick porous thermophones
}

Pierre Guiraud, Stefano Giordano, Olivier Bou Matar, Philippe Pernod, Raphael Lardat

\section{- To cite this version:}

Pierre Guiraud, Stefano Giordano, Olivier Bou Matar, Philippe Pernod, Raphael Lardat. Two temperature model for thermoacoustic sound generation in thick porous thermophones. Journal of Applied Physics, 2019, 126 (16), pp.165111. 10.1063/1.5121395 . hal-02337957

\section{HAL Id: hal-02337957 https://hal.science/hal-02337957}

Submitted on 24 Sep 2020

HAL is a multi-disciplinary open access archive for the deposit and dissemination of scientific research documents, whether they are published or not. The documents may come from teaching and research institutions in France or abroad, or from public or private research centers.
L'archive ouverte pluridisciplinaire HAL, est destinée au dépôt et à la diffusion de documents scientifiques de niveau recherche, publiés ou non, émanant des établissements d'enseignement et de recherche français ou étrangers, des laboratoires publics ou privés. 


\title{
Two temperature model for thermoacoustic sound generation in thick porous thermophones
}

\author{
Guiraud Pierre, ${ }^{1,2,3, \text { a) }}$ Giordano Stefano, ${ }^{1}$, b) Bou Matar Olivier, ${ }^{1}$ Pernod Philippe, ${ }^{1}$ and Lardat Raphael ${ }^{2}$ \\ 1) Univ. Lille, CNRS, Centrale Lille, ISEN, Univ. Valenciennes, UMR 8520 - IEMN, LIA LICS/LEMAC, F-59000 Lille, \\ France \\ ${ }^{2)}$ Thales Underwater System France, Sophia Antipolis, 525 Route des Dolines, F-06560 Valbonne, \\ France \\ ${ }^{3)}$ CINTRA UMI 3288, CNRS/NTU/THALES, Research Techno Plaza, 50 Nanyang Drive 637553, \\ Singapore
}

(Dated: 9 October 2019)

The thermoacoustic sound generation offers a promising wideband alternative to mechanically driven loudspeakers. Over the past decade, the development of nanomaterials with new physico-chemical properties promoted a wide interest in the thermophones technology. Indeed, several thermophone structures based on suspended nanowires, graphene sheets, highly porous foams or sponges have been investigated. At the same time, theoretical models have been developed to predict the frequency and power spectra of these devices. However, most of models have taken into consideration a solid homogeneous material for representing the thermophone generating layer, and its microstructure was therefore neglected. If this assumption holds for thin dense materials, it is not acceptable for thick and porous thermophone devices. Hence, a model able to describe the behavior of highly porous foam- or sponge-like generating layers is proposed. It is based on a two temperature scheme since the thermal equilibrium is not typically attained between the foam material and the embedded air. To do this, the fluid equations for the air are coupled with the heat equation for the solid foam through boundary conditions mimicking the energy exchange at the contact surface between them. The behavior of the main physical variables within the porous generating layer is explained and comparisons with recent experimental results are thoroughly discussed.

\section{INTRODUCTION}

For now more than a century, sound generation has been realized by means of electroacoustic transducers, which are able to convert an electrical signal into sound waves. In the classical scheme, a coil/magnet core induces the vibration of a membrane, eventually generating an acoustic wave. Also, piezoelectric materials are used for sound generation in specific situations, such as underwater applications or buzzer devices. For both magnetic and piezoelectric systems, the operating principle is based on mechanical vibrations generating sound. Despite the huge technological improvements of those technologies over the past century some limitations remains. Indeed, the mechanically based sound generation is a resonant phenomenon meaning that a single loudspeaker is not able to achieve a wideband response.

In the early 20th century, an alternative solution has been proposed by De Lange, ${ }^{1}$ Arnold \& Crandall, ${ }^{2}$ and Wente. ${ }^{3}$ They observed and theorized the thermoacoustic sound generation phenomenon. This is based on a material having a low heat capacity and a high thermal and electrical conductivity. Whenever an electrical oscillating current is applied to the sample, its temperature follows the current variations, and the air in the vicinity of the sample will experience compression and dilatation processes, thus generating a sound wave (see Fig.1). Due to the low efficiency of this principle and the limited availability of suitable materials, thermoacoustic sound generation was not largely exploited at that

\footnotetext{
a)Electronic mail: pierre.guiraud@centraliens-lille.org

b)Electronic mail: stefano.giordano@univ-lille.fr
}

time. More recently, an efficient thermophone device made of a thin aluminum film deposited on a porous silicon substrate has been realized and analyzed. ${ }^{4}$ From then on, the development of advanced nanotechnologies strongly promoted the rapid progress of the thermoacoustic applications. Indeed, over the past two decades many potential thermophones have been proposed and investigated. Without any pretension to being exhaustive, one could cite: thermophones with suspended nanowires (made of aluminum, ${ }^{5}$ gold, ${ }^{6}$ or silver $^{7}$ ), graphene thermophones (based on paper technology, ${ }^{8}$ foam structures, ${ }^{9}$ or laser scribing ${ }^{10}$ ) and multi walled nanotubes devices. ${ }^{11}$

The modeling of the thermoacoustic phenomena is important to analyse the behavior and design the structure of the thermophone systems. The original theory proposed by Arnold \& Crandall $^{2}$ was generalized in order to take into consideration the heat stored by the thermophone material. ${ }^{12}$ Later, energy based models have been used to describe the performances of thermophones deposited on a substrate. ${ }^{13,14}$ Also, the balance laws of continuum mechanics have been used to elaborate models for planar, ${ }^{15}$ cylindrical, ${ }^{16}$ and spherical thermophones. ${ }^{17}$ The same approach has been adopted to study the plane waves propagation in different thermophone configurations, such as the free field geometry, ${ }^{18}$ and the thermophone on substrate with an air gap. ${ }^{19}$ Lastly, a multilayer model taking the wave propagation in the solid thermophone layer into account has been proposed. ${ }^{20}$

In most models, the generating thermophone layer is considered as a homogeneous solid material. Consequently, the performances of the device are typically written in terms of the so-called heat capacity per unit area (HCPUA), which is defined as the product of the thickness of the layer $L_{l}$, its density $\rho_{s}$, and its heat capacity $C_{v, s}$. It is unanimously considered 


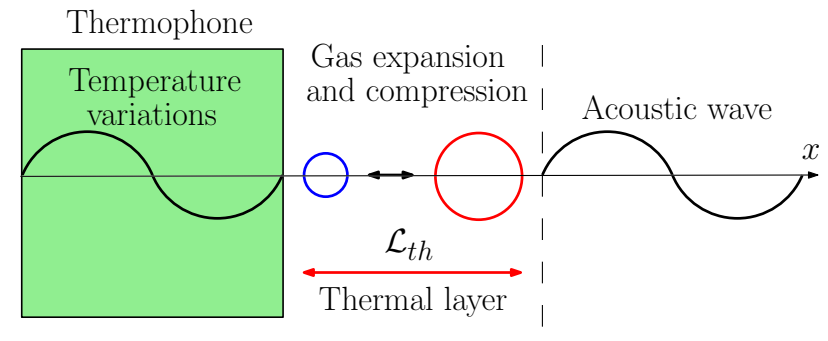

FIG. 1. Schematic of the thermoacoustic sound generation through thermophone devices.

as the most important parameter describing the thermophone performances. It should be strongly reduced for obtaining an efficient thermoacoustic generation. This principle is well verified for dense and thin film thermophones. However, new thick foam like materials assembled by chemical vapor deposition (CVD) are gaining interest because of their good efficiency and their higher mechanical stability. ${ }^{21}$ In spite of the interesting features of these materials, exhibiting a porosity larger than about $90 \%$, no theoretical model exists to accurately describe their behavior. Therefore, a two temperature model is presented here in order to gain a deeper insight into the thermoacoustic behavior of thermophones based on highly porous foam like materials. This model introduces the local non-equilibrium between the temperatures of the solid foam and the air embedded inside it. ${ }^{22-26}$ It means that two different temperatures are considered for each phase of the generating layer. The balance equations for the air are coupled with the heat equation for the foam through a set of boundary conditions describing the exchange of energy at each contact surface between air and foam. This is the most important difference between our model and previous ones: while in previous models, the heat exchange between generating layer and air occurs only at the two external surfaces, in the present case the actual distribution of air within the pores and the heat exchanges at any foam-air contact are considered. This process is able to explain the high efficiency observed in real thermophones based on foam- or sponge-like materials. A complete description of the main physical variable inside the generating layer is given together with a comparison between experimental and theoretical results for both thin film thermophones and thick porous thermophones.

The paper is structured as follows. In Section II, the two temperature theory can be found and a technique to solve the main equations in the case of a thermophone radiating planar waves in free field is proposed. In Section III, an acoustic diffraction theory, based on the classical Rayleigh's second integral, is introduced and used later on to draw a comparison with experimental results. In Section IV, the behavior of the most important physical variables is analyzed in order to clarify the thermoacoustic processes in a porous structure. Then, the two temperature model is compared with previous models and with experimental data taken from the recent literature. For the sake of completeness, the models are applied to both thin film thermophones and thick porous thermophones.

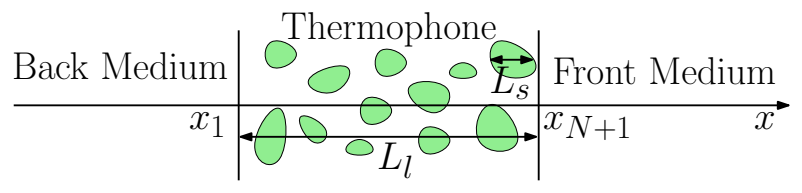

FIG. 2. Schematic of a porous thermophone radiating in free field (symmetrically in back and front media). The thermophone layer has a width $L_{l}$ and foam branches of average diameter $L_{s}$. The volumetric source $S_{0}$ is supplied to the foam structure by Joule effect.

\section{TWO TEMPERATURE THEORETICAL MODEL FOR A THERMOPHONE IN FREE FIELD}

The general conservation equations for mass, linear momentum and energy in a fluid medium can be written as ${ }^{27}$

$$
\begin{array}{r}
\frac{1}{B} \frac{\partial p}{\partial t}-\alpha_{T} \frac{\partial T}{\partial t}+\vec{\nabla} \cdot \vec{v}=0 \\
\rho \frac{\partial \vec{v}}{\partial t}=-\vec{\nabla} p+\mu \nabla^{2} \vec{v}+(\lambda+\mu) \vec{\nabla}(\vec{\nabla} \cdot \vec{v}) \\
\rho C_{p} \frac{\partial T}{\partial t}-\alpha_{T} T_{0} \frac{\partial p}{\partial t}=\kappa \nabla^{2} T
\end{array}
$$

where the pressure $p(\vec{r}, t)[\mathrm{Pa}]$, the temperature variation $T(\vec{r}, t)[\mathrm{K}]$ and the particle velocity vector $\vec{v}(\vec{r}, t)[\mathrm{m} / \mathrm{s}]$ are the main variables depending on time $t[\mathrm{~s}]$ and position vector $\vec{r}$ $[\mathrm{m}]$. Moreover, $\rho$ is the density $\left[\mathrm{kg} / \mathrm{m}^{3}\right], B$ the Bulk Modulus $[\mathrm{Pa}], \alpha_{T}$ the coefficient of volumetric expansion $[1 / \mathrm{K}]$, $\lambda$ and $\mu$ the first and second viscosity coefficients [Pa.s], $C_{p}$ the specific heat at constant pressure $[\mathrm{J} /(\mathrm{kg} \cdot \mathrm{K})], T_{0}$ the ambient temperature $[\mathrm{K}]$ and, finally, $\kappa$ the thermal conductivity $[\mathrm{W} /(\mathrm{m} \cdot \mathrm{K})]$. All these parameters will be considered as constants for a given fluid medium. These equations represent the linearized motion of the fluid around the equilibrium state identified by $T=T_{0}, p=p_{0}$ and $\vec{v}=0$.

To begin, a thermophone radiating in free field is considered, as shown in Fig.2. The central active layer of the thermophone is assumed to be made of a porous material with a very high porosity (e.g., larger than 90\%), with an undeformable solid microstructure. Therefore, a model is elaborated to take into account the effect of the fluid (typically air) embedded within the pores of the active solid phase, i.e. the coupling between the fluid and the micro- or nano-structured porous solid in the active region. The fluid motion within the pores of the thermophone region can be described by Eqs.(1)-(3). However, due to the possible high frequency of the electric energy supplied, the local thermal equilibrium is not attained between fluid and solid phases. Therefore, the conservation equations in the fluid can be coupled with the energy conservation for the solid phase, where an input power density $S_{0}$ is introduced (Joule effect), and a temperature $T_{s} \neq T$ is considered. The combination of such equations represents the non thermal equilibrium between fluid and solid. In other terms, since the temperatures of fluid and solid are sensibly different on the two sides of a real contact interface, a phenomenological two-temperature model has to be considered to effectively take account of this non thermal equilibrium. Moreover, to better take into consideration the morphology of the porous 


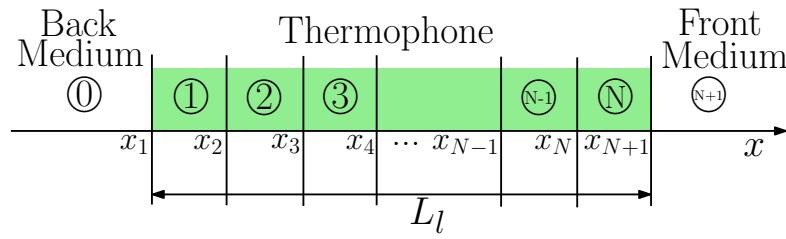

FIG. 3. Two-temperature representation of the porous thermophone. In each layer, a temperature $T$ is associated to the air within the pores, and a temperature $T_{s} \neq T$ is associated to the foam branches. The interfaces control the energy exchange between foam and air.

structure within the generation layer, an exchange of energy at the contact surfaces between pores and foam branches is introduced. This scheme can be mimicked by a series of interfaces, which are represented in Fig.3. Each layer is therefore described by the following set of equations

$$
\begin{array}{r}
\frac{1}{B} \frac{\partial p}{\partial t}-\alpha_{T} \frac{\partial T}{\partial t}+\vec{\nabla} \cdot \vec{v}=0, \\
\rho \frac{\partial \vec{v}}{\partial t}=-\vec{\nabla} p+\mu \nabla^{2} \vec{v}+(\lambda+\mu) \vec{\nabla}(\vec{\nabla} \cdot \vec{v}), \\
\rho C_{p} \frac{\partial T}{\partial t}-\alpha_{T} T_{0} \frac{\partial p}{\partial t}=\kappa \nabla^{2} T, \\
\rho_{s} C_{v, s} \frac{\partial T_{s}}{\partial t}=\kappa_{s} \nabla^{2} T_{s}+S_{0},
\end{array}
$$

where the last equation represent the added energy conservation in the solid foam and the subscript $s$ is used for the solid/foam parameters. For instance, $C_{v, s}$ is the specific heat at constant volume of the foam $[\mathrm{J} /(\mathrm{kg} \cdot \mathrm{K})]$. For the sake of simplicity, this system is investigated in the one-dimensional case (plane wave propagation along the $x$ axis) with an harmonic time dependence. Then, one gets

$$
\begin{array}{r}
\frac{\mathrm{i} \omega}{B} p-\mathrm{i} \omega \alpha_{T} T+\frac{\mathrm{d} v}{\mathrm{~d} x}=0, \\
\mathrm{i} \omega \rho v=-\frac{\mathrm{d} p}{\mathrm{~d} x}+(\lambda+2 \mu) \frac{\mathrm{d}^{2} v}{\mathrm{~d} x^{2}}, \\
\mathrm{i} \omega \rho C_{p} T=\kappa \frac{\mathrm{d}^{2} T}{\mathrm{~d} x^{2}}+\mathrm{i} \omega \alpha_{T} T_{0} p, \\
\mathrm{i} \omega \rho_{s} C_{v, s} T_{s}=\kappa_{s} \frac{\mathrm{d}^{2} T_{s}}{\mathrm{~d} x^{2}}+S_{0},
\end{array}
$$

where $i$ is the imaginary unit. Importantly, in these equations, the terms $\rho_{s} C_{v, s}$ and $\kappa_{s}$ must be considered as the homogenized values over the whole volume of the generation layer. Indeed, the two temperature model implicitly considers two effective phases (air and foam, in our case), both occupying the whole region of the thermophone layer. Therefore, for each phase the homogenized values for all physical properties have to be considered. ${ }^{22-26}$ However, since the materials display a very high porosity, the homogenized parameters are adopted only for the foam phase.

The energy exchange between air in the pores and solid branches is written in the boundary conditions that are summarized below. To write these conditions, the definition of the heat flux is introduced in the air phase as $q=-\kappa \mathrm{d} T / \mathrm{d} x$ and in the solid foam phase as $q_{s}=-\kappa_{s} \mathrm{~d} T_{s} / \mathrm{d} x$. Moreover, instead of considering the pressure $p$, the normal surface tension $\tilde{p}$ is adopted as $\tilde{p}=p-(\lambda+2 \mu) \mathrm{d} v / \mathrm{d} x$, which takes into account the viscous stress. Now, in each layer of the thermophone (layers from 1 to $N$, see Fig.3), there are four variables $\tilde{p}, v, T$ and $q$ for the air and two variables $T_{s}$ and $q_{s}$ for the branches. On the other hand, in the back and front media (layers 0 and $N+1$ ), there are only the variables for the air, namely $\tilde{p}, v, T$ and $q$. An index $0 \leq i \leq N+1$ is associated to these variables to identify the layer where they are defined. For the interfaces within the thermophone (from $x_{2}$ to $x_{N}$ ), one has

$$
\begin{aligned}
\tilde{p}_{i-1}\left(x_{i}\right) & =\tilde{p}_{i}\left(x_{i}\right), \\
v_{i-1}\left(x_{i}\right) & =v_{i}\left(x_{i}\right), \\
T_{i-1}\left(x_{i}\right) & =T_{i}\left(x_{i}\right), \\
T_{s, i-1}\left(x_{i}\right) & =T_{s, i}\left(x_{i}\right), \\
q_{i}\left(x_{i}\right) & =q_{i-1}\left(x_{i}\right)+g\left(T_{s, i}\left(x_{i}\right)-T_{i}\left(x_{i}\right)\right), \\
q_{s, i}\left(x_{i}\right) & =q_{s, i-1}\left(x_{i}\right)-g\left(T_{s, i}\left(x_{i}\right)-T_{i}\left(x_{i}\right)\right),
\end{aligned}
$$

for all $i=2, \ldots, N$. While the first four relations represent the classical continuity of normal stress, velocity and temperatures, the last two relations represent the energy exchanged between solid branches and air. Indeed, if $T_{s, i}\left(x_{i}\right) \geq T_{i}\left(x_{i}\right)$ a positive quantity of energy leaves the foam and moves to the surrounding air. This process is controlled by a new parameter $g\left[\mathrm{~W} /\left(\mathrm{m}^{2} \cdot \mathrm{K}\right)\right]$, describing the fluid/solid coupling at the interfaces. Concerning the first interface at $x_{1}$ (back medium/thermophone), the result is

$$
\begin{aligned}
\tilde{p}_{0}\left(x_{1}\right) & =\tilde{p}_{1}\left(x_{1}\right), \\
v_{0}\left(x_{1}\right) & =v_{1}\left(x_{1}\right), \\
T_{0}\left(x_{1}\right) & =T_{1}\left(x_{1}\right), \\
q_{1}\left(x_{1}\right) & =q_{0}\left(x_{1}\right)+g\left(T_{s, 1}\left(x_{1}\right)-T_{1}\left(x_{1}\right)\right), \\
q_{s, 1}\left(x_{1}\right) & =-g\left(T_{s, 1}\left(x_{1}\right)-T_{i}\left(x_{1}\right)\right),
\end{aligned}
$$

and, finally the last interface equation at $x_{N+1}$ (thermophone/front medium) is written as

$$
\begin{aligned}
\tilde{p}_{N}\left(x_{N+1}\right) & =\tilde{p}_{N+1}\left(x_{N+1}\right), \\
v_{N}\left(x_{N+1}\right) & =v_{N+1}\left(x_{N+1}\right), \\
T_{N}\left(x_{N+1}\right) & =T_{N+1}\left(x_{N+1}\right), \\
q_{N+1}\left(x_{N+1}\right) & =q_{N}\left(x_{N+1}\right)+g\left(T_{S, N}\left(x_{N+1}\right)-T_{N}\left(x_{N+1}\right)\right), \\
0 & =q_{s, N}\left(x_{N+1}\right)-g\left(T_{s, N}\left(x_{N+1}\right)-T_{N}\left(x_{N+1}\right)\right) .
\end{aligned}
$$

It is interesting to observe that this proposed two-temperature model differs form the classical two-temperature model described in the literature. ${ }^{22-26}$ Indeed, in our case the exchange of energy is confined at all interfaces representing the contacts between air and foam. This process is not continuous and therefore it is not implemented within the balance equations defined in each layer (see Eqs.(8)-(11)). On the other hand, it is important to also note that in this thermophone model the heating of the air is a process distributed (although not continuously) within the whole region of the generation layer (at all the air/foam contacts) and not only at the external interfaces (at $x_{1}$ and $x_{N+1}$ ) as in the classical thermophone models. ${ }^{20}$ For this reason, it is able to represent the behavior of thick and porous thermophone devices. 
The solution for the physical variables defined in each layer of the model can be found as follows. From Eq.(8) the pressure can be obtained as

$$
p=\alpha_{T} B T-\frac{B}{\mathrm{i} \omega} \frac{\mathrm{d} v}{\mathrm{~d} x} .
$$

Then, the normal surface tension $\tilde{p}$ becomes

$$
\tilde{p}=\alpha_{T} B T-\left(\lambda+2 \mu+\frac{B}{\mathrm{i} \omega}\right) \frac{\mathrm{d} v}{\mathrm{~d} x} .
$$

Using Eq.(29) along with Eqs.(8)-(11), the velocity is eventually found as

$$
\begin{aligned}
v & =-\frac{1}{\mathrm{i} \omega \rho}\left[\alpha_{T} B+\frac{\mathrm{i} \omega \rho C_{v}}{\alpha_{T} T_{0} B}\left(\lambda+2 \mu+\frac{B}{\mathrm{i} \omega}\right)\right] \frac{\mathrm{d} T}{\mathrm{~d} x} \\
& +\left(\lambda+2 \mu+\frac{B}{\mathrm{i} \omega}\right) \frac{\kappa}{\mathrm{i} \omega \rho \alpha_{T} T_{0} B} \frac{\mathrm{d}^{3} T}{\mathrm{~d} x^{3}},
\end{aligned}
$$

where the thermodynamic relationship between the specific heats $\rho\left(C_{p}-C_{v}\right)=T_{0} \alpha_{T}^{2} B$ has been used. The fourth order differential equation for $T$ can be then found in the form

$$
\begin{aligned}
0 & =\left(\lambda+2 \mu+\frac{B}{\mathrm{i} \omega}\right) \kappa \frac{\mathrm{d}^{4} T}{\mathrm{~d} x^{4}}-\omega^{2} \rho^{2} C_{v} T \\
& -\left[\left(\lambda+2 \mu+\frac{B}{\mathrm{i} \omega}\right) \mathrm{i} \omega \rho C_{v}+\left(\mathrm{i} \omega \rho \kappa+\alpha_{T}^{2} T_{0} B^{2}\right)\right] \frac{\mathrm{d}^{2} T}{\mathrm{~d} x^{2}}
\end{aligned}
$$

coherently with recent investigations. ${ }^{20}$ The solutions of Eq.(31) represent thermal modes and acoustical modes that will be described by $\theta_{t h}$ and $k_{a c}$, respectively. The temperature in the fluid can be therefore written as

$$
T=A e^{-\mathrm{i} k_{a c} x}+B e^{+\mathrm{i} k_{a c} x}+C e^{-\theta_{t h} x}+D e^{+\theta_{t h} x} .
$$

On the other hand, from the energy conservation in the solid, Eq.(11), the temperature $T_{s}$ can be easily written as

$$
T_{s}=E e^{-\theta_{\text {solid }} x}+F e^{+\theta_{\text {solid }} x}+T_{s, 0},
$$

with $T_{s, 0}$ being the particular solution of Eq.(11) given by

$$
T_{s, 0}=\frac{S_{0}}{\rho_{s} C_{v, s} \mathrm{i} \omega} .
$$

In previous solutions $A, B, C, D, E$ and $F$ are constants to be determined. In order to obtain the values of $\theta_{t h}$ and $k_{a c}$ the following parameters are introduced

$$
\begin{array}{r}
\gamma=\frac{C_{p}}{C_{v}}, \\
C_{0}=\sqrt{\frac{B}{\rho} \gamma} \\
l_{k}=\frac{C_{0} \kappa}{B C_{p}}, \\
l_{v}=\frac{\lambda+2 \mu}{\rho C_{0}} .
\end{array}
$$

In order to find the values of $k_{a c}$ and $\theta_{t h}$ one should find the solutions of the algebraic fourth-order (biquadratic) characteristic equation associated to Eq.(31). Since the exact solutions are rather cumbersome, it is useful to assume a weak thermal conduction and a weak viscosity of the adopted medium. Under these hypotheses, the asymptotic solutions of Eq.(31) can be obtained in the form

$$
\begin{aligned}
& k_{a c}= \pm \frac{\omega}{C_{0}}\left[1-\frac{1}{2} \frac{i \omega}{C_{0}} l_{v}-\frac{1}{2} \frac{i \omega}{C_{0}} l_{k}\left(1-\frac{1}{\gamma}\right)\right] \\
& \theta_{t h}= \pm \sqrt{\frac{i \omega \gamma}{C_{0} l_{k}}}\left[1+\frac{1}{2} \frac{i \omega}{C_{0}} l_{k}\left(1-\frac{1}{\gamma}\right)+\frac{1}{2} \frac{i \omega}{C_{0}} l_{v}(1-\gamma)\right] .
\end{aligned}
$$

While the detailed proof of Eqs.(39) and (40) is given in the recent literature ${ }^{20}$ it is worth noticing that these results represent the first order expansions (with small $\kappa, \lambda$ and $\mu$ ) of the solutions of the algebraic equation associated with Eq.(31). For the air this is a good approximation in the frequency range of interest for the thermophone applications. If necessary, the values of $\theta_{t h}$ and $k_{a c}$ can be obtained numerically, without any assumption on the material parameters. On the other hand, through Eq.(11), the propagation constant in the solid can also be found as

$$
\theta_{\text {solid }}= \pm \sqrt{\frac{\mathrm{i} \omega \rho_{s} C_{v, s}}{\kappa_{s}}}
$$

The set of solutions in a layer, concerning the air variables, is obtained as

$$
\begin{aligned}
\tilde{p}= & A \mathscr{F}\left(-\mathrm{i} k_{a c}\right) \mathrm{e}^{-\mathrm{i} k_{a c} x}+B \mathscr{F}\left(\mathrm{i} k_{a c}\right) \mathrm{e}^{\mathrm{i} k_{a c} x} \\
& +C \mathscr{F}\left(-\theta_{t h}\right) \mathrm{e}^{-\theta_{t h} x}+D \mathscr{F}\left(\theta_{t h}\right) \mathrm{e}^{\theta_{t h} x}, \\
v= & A \mathscr{G}\left(-\mathrm{i} k_{a c}\right) \mathrm{e}^{-\mathrm{i} k_{a c} x}+B \mathscr{G}\left(\mathrm{i} k_{a c}\right) \mathrm{e}^{\mathrm{i} k_{a c} x} \\
& +C \mathscr{G}\left(-\theta_{t h}\right) \mathrm{e}^{-\theta_{t h} x}+D \mathscr{G}\left(\theta_{t h}\right) \mathrm{e}^{\theta_{t h} x}, \\
q= & A \kappa \mathrm{i} k_{a c} \mathrm{e}^{-\mathrm{i} k_{a c} x}-B \kappa \mathrm{i} k_{a c} \mathrm{e}^{\mathrm{i} k_{a c} x} \\
& +C \kappa \theta_{t h} \mathrm{e}^{-\theta_{t h} x}-D \kappa \theta_{t h} \mathrm{e}^{\theta_{t h} x}, \\
T= & A \mathrm{e}^{-\mathrm{i} k_{a c} x}+B \mathrm{e}^{\mathrm{i} k_{a c} x}+C \mathrm{e}^{-\theta_{t h} x}+D \mathrm{e}^{\theta_{t h} x},
\end{aligned}
$$

where the functions

$$
\begin{aligned}
& \mathscr{F}(\eta)=\alpha_{T} B-\left(\frac{B}{i \omega}+\lambda+2 \mu\right)\left(\mathrm{L}_{1} \eta^{2}+\mathrm{L}_{2} \eta^{4}\right) \\
& \mathscr{G}(\eta)=\mathrm{L}_{1} \eta+\mathrm{L}_{2} \eta^{3}
\end{aligned}
$$

have been introduced, with $L_{1}$ and $L_{2}$ being the coefficients (see Eq.(30))

$$
\begin{aligned}
& \mathrm{L}_{1}=-\frac{1}{\mathrm{i} \omega \rho}\left[\alpha_{T} B+\frac{\mathrm{i} \omega \rho C_{v}}{\alpha_{T} T_{0} B}\left(\lambda+2 \mu-\mathrm{i} \frac{B}{\omega}\right)\right], \\
& \mathrm{L}_{2}=\left(\lambda+2 \mu-\mathrm{i} \frac{B}{\omega}\right) \frac{\kappa}{\mathrm{i} \omega \rho \alpha_{T} T_{0} B} .
\end{aligned}
$$

By defining the matrices

$$
H^{(a)}=\left[\begin{array}{llll}
\mathscr{F}\left(-\mathrm{i} k_{a c}\right) & \mathscr{F}\left(\mathrm{i} k_{a c}\right) & \mathscr{F}\left(-\theta_{t h}\right) & \mathscr{F}\left(\theta_{t h}\right) \\
\mathscr{G}\left(-\mathrm{i} k_{a c}\right) & \mathscr{G}\left(\mathrm{i} k_{a c}\right) & \mathscr{G}\left(-\theta_{t h}\right) & \mathscr{G}\left(\theta_{t h}\right) \\
\kappa \mathrm{i} k_{a c} & -\kappa \mathrm{i} k_{a c} & \kappa \theta_{t h} & -\kappa \theta_{t h} \\
1 & 1 & 1 & 1
\end{array}\right]
$$


and

$$
H^{(b)}(x)=\left[\begin{array}{llll}
\mathrm{e}^{-\mathrm{i} k_{a c} x} & 0 & 0 & 0 \\
0 & \mathrm{e}^{\mathrm{i} k_{a c} x} & 0 & 0 \\
0 & 0 & \mathrm{e}^{-\theta_{t h} x} & 0 \\
0 & 0 & 0 & \mathrm{e}^{\theta_{t h} x}
\end{array}\right],
$$

the general solution in a given fluid layer is given by

$$
\left[\begin{array}{c}
\tilde{p}_{i}(x) \\
v_{i}(x) \\
q_{i}(x) \\
T_{i}(x)
\end{array}\right]=H^{(a)} H^{(b)}(x)\left[\begin{array}{c}
A_{i} \\
B_{i} \\
C_{i} \\
D_{i}
\end{array}\right],
$$

for all $0 \leq i \leq N+1$. It is important to observe that the relations $A_{0}=C_{0}=B_{N+1}=D_{N+1}=0$ must be imposed since no progressive wave in the back medium (identified by $i=0$ ) and no regressive wave in the front medium (identified by $i=N+1$ ) are considered. Similarly, the general solution for the solid/foam variables in a given layer is given by

$$
\left[\begin{array}{l}
q_{s, i}(x) \\
T_{s, i}(x)
\end{array}\right]=G(x)\left[\begin{array}{c}
E_{i} \\
F_{i}
\end{array}\right]+\left[\begin{array}{c}
0 \\
T_{s, 0}
\end{array}\right]
$$

for all $1 \leq i \leq N$. Here, the matrix

$$
G(x)=\left[\begin{array}{cc}
\kappa_{s} \theta_{\text {solid }} e^{-\theta_{\text {solid }} x} & -\kappa_{s} \theta_{\text {solid }} e^{\theta_{\text {solid }} x} \\
e^{-\theta_{\text {solid }} x} & e^{\theta_{\text {solid }} x}
\end{array}\right],
$$

is introduced. The knowledge of the complete solution of the problem for all the physical variables and all the layers (see Eqs.(52) and (53)) allows to implement the boundary conditions given in Eqs.(12)-(27). Indeed, it is not difficult to prove that these conditions are represented by $6 N+4$ linear equations, with exactly $6 N+4$ unknown coefficients. The system is well posed and can therefore always be solved by standard numerical methods.

Interestingly enough, the model stated in this Section can be further improved by adding a term corresponding to the heat loss at the interfaces, which is described by a new coefficient $\beta_{s}\left[\mathrm{~W} /\left(\mathrm{m}^{2} \mathrm{~K}\right)\right]$. To introduce this effect, Eqs.(16),(21) and (26) must be substituted with

$$
\begin{aligned}
q_{i}\left(x_{i}\right)= & q_{i-1}\left(x_{i}\right)+g\left(T_{s, i}\left(x_{i}\right)-T_{i}\left(x_{i}\right)\right)-\beta_{s} T_{i}\left(x_{i}\right), \\
q_{1}\left(x_{1}\right)= & q_{0}\left(x_{1}\right)+g\left(T_{s, 1}\left(x_{1}\right)-T_{1}\left(x_{1}\right)\right)-\beta_{s} T_{1}\left(x_{1}\right), \\
q_{N+1}\left(x_{N+1}\right)= & q_{N}\left(x_{N+1}\right)+g\left(T_{s, N}\left(x_{N+1}\right)-T_{N}\left(x_{N+1}\right)\right) \\
& -\beta_{S} T_{N}\left(x_{N+1}\right),
\end{aligned}
$$

where the last term in each relation, described by the coefficient $\beta_{s}$, models the influence of conduction, convection, and radiation losses. The total loss is considered proportional to the temperature and this represents a good approximation for losses due to conduction and convection. However, it is a poor representation of the radiated heat, which is typically proportional to the fourth power of the temperature. Nevertheless, the thermal losses by radiation in a thermophone are negligible in most of the cases. ${ }^{28}$ More general approaches for considering imperfect interfaces can be found in the literature. ${ }^{29,30}$

\section{ACOUSTIC DIFFRACTION THEORY}

The model here developed describes the thermo-acoustic sound generation for a thermophone with a infinitely large surface, radiating in near field. However, real measurements are typically done in far field, with finite size thermophones. Therefore, an acoustic diffraction theory must be implemented to compare the results of our model with experiments. If a planar surface at $x=x_{0}$, vibrating with the velocity $v(y, z)$, is studied, each point can be considered as an acoustic source. Hence, the actual pressure field can be found by superposition, using the classical Rayleigh's second integral

$$
p_{\mathrm{FF}}(x, y, z)=\frac{\mathrm{i} \omega \rho}{4 \pi} \int_{-L_{y}}^{L_{y}} \int_{-L_{z}}^{L_{z}} v\left(y^{\prime}, z^{\prime}\right) \frac{e^{-\mathrm{i} k r}}{r} d z^{\prime} d y^{\prime},
$$

where $r=\sqrt{\left(x-x_{0}\right)^{2}+\left(y-y^{\prime}\right)^{2}+\left(z-z^{\prime}\right)^{2}}$ is the distance between generation and observation points, with $(x, y, z)$ the coordinates of the observation point and $\left(x_{0}, y^{\prime}, z^{\prime}\right)$ those of the generation point. The rectangle $\left(-L_{y}, L_{y}\right) \times\left(-L_{z}, L_{z}\right)$ represents the vibrating region of the plane. In order to use Eq.(58), the velocity field in air is calculated through our near field model for a distance from the thermophone slightly larger than the thickness of the thermal layer $\mathscr{L}_{t h}$ (active region), as defined in Fig.1. Indeed, at this distance, the velocity has attained its maximum value, which can be considered for the acoustic propagation in Eq.(58). The thickness of the thermal layer has been evaluated through the approximated expression $\mathscr{L}_{t h}=2 \sqrt{\frac{C_{0} l_{k}}{2 \omega \gamma}}{ }^{20}$ Hence, the theoretical curves that will be shown in Section IV represents the results of the Rayleigh's second integral applied to the velocity field of our near field model. In other words, our model is used to describe the thermoacoustic generation of waves whereas the diffraction theory is used to properly take into account the resulting acoustic propagation.

\section{ANALYSIS AND COMPARISON WITH EXPERIMENTAL RESULTS}

In Section II, a two temperature model for the thermoacoustic sound generation has been elaborated. This model was motivated by the fact that most of the currently used thermophones are composed of non continuous materials. For instance, widely adopted structures are based on multi walled nanotubes (MWNT), arranged as sheets, forests, foams or sponges. ${ }^{31}$ Those thermophones devices are therefore made of both air and nanotubes. Thus, the models based on a continuous homogeneous medium do not represent the real microstructure of these systems and neglect the interaction fluid/solid in the active region. As a matter of fact, in foam like thermophones a porosity of the active layer as large as 99\% can be achieved, making the air component not negligible.

In the following (see Section IV A), the thermo-acoustically generated temperatures, particle velocity and heat fluxes are analyzed as a function of the position $x$ (one-dimensional modeling) at a frequency of $3 \mathrm{kHz}$. This allows for a deeper 


\begin{tabular}{c|cccccccc} 
& $\begin{array}{c}\rho \\
{\left[\mathrm{kg} \cdot \mathrm{m}^{-3}\right]}\end{array}$ & $\begin{array}{c}C_{p} \\
{\left[\mathrm{~J} \cdot \mathrm{kg}^{-1} \cdot \mathrm{K}^{-1}\right]}\end{array}$ & $\begin{array}{c}C_{V} \\
{\left[\mathrm{~J} \cdot \mathrm{kg}^{-1} \cdot \mathrm{K}^{-1}\right]}\end{array}$ & $\begin{array}{c}B \\
{[\mathrm{~Pa}]}\end{array}$ & $\begin{array}{c}\alpha_{T} \\
{\left[\mathrm{~K}^{-1}\right]}\end{array}$ & $\begin{array}{c}\kappa \\
{\left[\mathrm{W} \cdot \mathrm{K}^{-1} \cdot \mathrm{m}^{-1}\right]}\end{array}$ & $\begin{array}{c}\lambda \\
{\left[\mathrm{N} \cdot \mathrm{s} \cdot \mathrm{m}^{-2}\right]}\end{array}$ & $\begin{array}{c}\mu \\
{\left[\mathrm{N} \cdot \mathrm{s} \cdot \mathrm{m}^{-2}\right]}\end{array}$ \\
\hline Gas, air & 1.20 & $9.96 \times 10^{2}$ & $7.17 \times 10^{2}$ & $1.01 \times 10^{5}$ & $3.33 \times 10^{-3}$ & $26.2 \times 10^{-3}$ & $16.82 \times 10^{-6}$ & $5.61 \times 10^{-6}$
\end{tabular}

TABLE I. Parameters of the propagating medium (air).

\begin{tabular}{l|ccccccc} 
& $\begin{array}{c}\rho_{s} \\
{\left[\mathrm{~kg} \cdot \mathrm{m}^{-3}\right]}\end{array}$ & $\begin{array}{c}C_{V, s} \\
{\left[\mathrm{~J} \cdot \mathrm{kg}^{-1} \cdot \mathrm{K}^{-1}\right]}\end{array}$ & $\begin{array}{c}B_{s} \\
{[\mathrm{~Pa}]}\end{array}$ & $\begin{array}{c}\kappa_{s} \\
{\left[\mathrm{~W} \cdot \mathrm{K}^{-1} \cdot \mathrm{m}^{-1}\right]}\end{array}$ & $\begin{array}{c}L_{l} \\
{[\mathrm{~m}]}\end{array}$ & $\begin{array}{c}L_{s} \\
{[\mathrm{~m}]}\end{array}$ & $\begin{array}{c}A \\
{[\mathrm{~cm} \times \mathrm{cm}]}\end{array}$ \\
\hline CNT sheet & 1 & 716 & $1.11 \times 10^{11}$ & 50 & $18 \times 10^{-6}$ & $10 \times 10^{-9}$ & Square $1.5 \times 1.5$ \\
ITO PAN & 220 & 606 & $1.65 \times 10^{11}$ & 310 & $5 \times 10^{-6}$ & $450 \times 10^{-9}$ & Square $1.2 \times 1.5$ \\
MWNT sponge & 30 & 716 & $1.11 \times 10^{11}$ & 100 & $100 \times 10^{-6}$ & $10 \times 10^{-9}$ & Square $1 \times 1$ \\
Graphene sponge & 2.75 & 660 & $1.44 \times 10^{8}$ & 6.3 & $800 \times 10^{-6}$ & $5 \times 10^{-9}$ & Circle $d=1.8$
\end{tabular}

TABLE II. Parameters of the thermophone materials used in the experiments and theoretical models. ${ }^{31}$

understanding of the model and a better interpretation of the thermoacoustic generation phenomena in porous devices.

Additionally, measurements published in the literature will be compared to the theoretical response of our two temperature model (referred to as $2 \mathrm{~T}$ model from now on) and of the classical model based on a homogeneous and solid active layer (referred to as 1T model from now on). ${ }^{20}$ The comparison will be discussed in two distinct sections, dealing with different thickness of the thermophone. Section IV B will investigate thin film thermophones with thickness of a few microns, and Section IV C foam like thermophones with thickness of hundreds of microns. The experimental data are taken from a recent investigation. ${ }^{31}$ This work provides frequency and power spectra of a wide variety of samples with a full description of the experimental setup. Thermal parameters of the samples were also measured and discussed. These data have been used in the theoretical models, making the comparison theory/experiment legitimate.

All of the presented theoretical results have been obtained using the parameters in Table I and Table II. A specific discussion concerns the parameter $g$, which is the only new parameter introduced in this model. The real total contact area between solid foam and air is given by $a \rho_{s} V$, where $a$ is the specific area of the foam $\left[\mathrm{m}^{2} / \mathrm{kg}\right], \rho_{s}$ is the foam density $\left[\mathrm{kg} / \mathrm{m}^{3}\right]$ and $V$ is the total foam volume $\left[\mathrm{m}^{3}\right]$ (of course, $a \rho_{s}$ is the interfacial area per unit volume). On the other hand, the effective contact area introduced in our model is given by $N S$, where $N$ is the number of layers in Fig.2, and $S$ is their area. On the basis of these premises, $g$ can be defined as $N S g=a \rho_{s} V h$, where $h$ is the real heat film transfer coefficient $\left[\mathrm{W} /\left(\mathrm{m}^{2} \cdot \mathrm{K}\right)\right]$ (convective and radiative). One simply gets $g=\frac{a \rho_{s} V h}{N S}=\frac{a \rho_{s} h L_{l}}{N}$, where $L_{l}=V / S$. With the reasonable values $a=1000-2000 \mathrm{~m}^{2} / \mathrm{g},{ }^{32,33} h=200 \mathrm{~W} /\left(\mathrm{m}^{2} \cdot \mathrm{K}\right),{ }^{34}$ $\rho_{s}=30 \mathrm{~kg} / \mathrm{m}^{3}, L_{l}=0.1-1 \mathrm{~mm}$, and $N=10$, one obtains $g \cong 10^{5} \mathrm{~W} /\left(\mathrm{m}^{2} \cdot \mathrm{K}\right)$, which is used throughout all the paper.

The $2 \mathrm{~T}$ model and the classical $1 \mathrm{~T}$ model, ${ }^{20}$ based on a single homogeneous active layer, have been implemented (see Fig.2). Both models have been adapted for acoustic diffraction as discussed in Section III, by considering the size and the shape of the samples used in the experimental activity. ${ }^{31}$ All results show the sound pressure level (SPL), defined in decibels $(\mathrm{dB})$, and calculated as

$$
\mathrm{SPL}=20 \log _{10}\left(\frac{p_{\mathrm{rms}}}{p_{\text {ref }}}\right),
$$

where $p_{\mathrm{rms}}$ is the root mean square pressure (i.e. $\left|p_{\mathrm{FF}}\right| / \sqrt{2}$, where $p_{\mathrm{FF}}$ is the complex pressure introduced in previous sections) and $p_{\text {ref }}$ is the reference sound pressure being, by definition, $20 \mu \mathrm{Pa}$ in air. The results are shown at a distance of $3 \mathrm{~cm}$ from the thermophone. The frequency spectrum results are normalized with power and the power spectrum results are shown at $3 \mathrm{kHz}$.

\section{A. Behavior of the physical variables with the two temperature model}

Here, the behavior of the main physical variables is shown by comparing a thick and a thin thermophone. For the thick thermophone the parameters of the graphene sponge $\left(L_{l}=\right.$ $800 \times 10^{-6} \mathrm{~m}$ ) are adopted, while for the thin one the parameters of the CNT sheet $\left(L_{l}=18 \times 10^{-6} \mathrm{~m}\right)$ are used. In Fig.4, one can find the temperatures, the particle velocity and heat fluxes versus $x$, calculated with the $1 \mathrm{~T}$ and the $2 \mathrm{~T}$ models for both the thick and thin thermophones at $f=3 \mathrm{kHz}$. In this figure, the curves corresponding to the absolute value of the different complex quantities have been plotted. To better explain the behavior of the heat flux within the system, in Fig.5, the real and imaginary parts of the heat fluxes in air and foam are shown as well. The input power is the same for all curves (1W) in Figs.4 and 5. The geometry considered is shown in Fig.3, where $N=5$ is imposed to easily identify the pores/branches structure in the plots. Moreover, the diffraction procedure is not implemented to obtain the results of this Section since only the near field generation is investigated. Please note that the 1T model gives the behavior of the physical quantities only outside the thermophone layer. On the contrary, the $2 \mathrm{~T}$ model allows for the complete analysis, also in the generating porous structure.

Fig.4 (panels a and b) shows that despite a slightly higher value of the temperature on the edges of the thermophone for the 1T model, both thick and thin systems display the same behavior in air. The thermal active layer $\mathscr{L}_{t h}$ can be seen in 
(a)

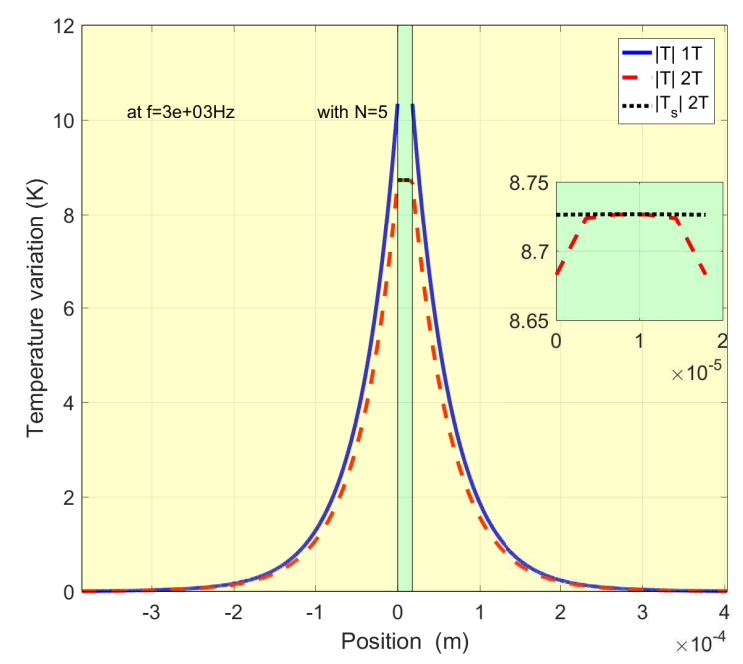

(c)

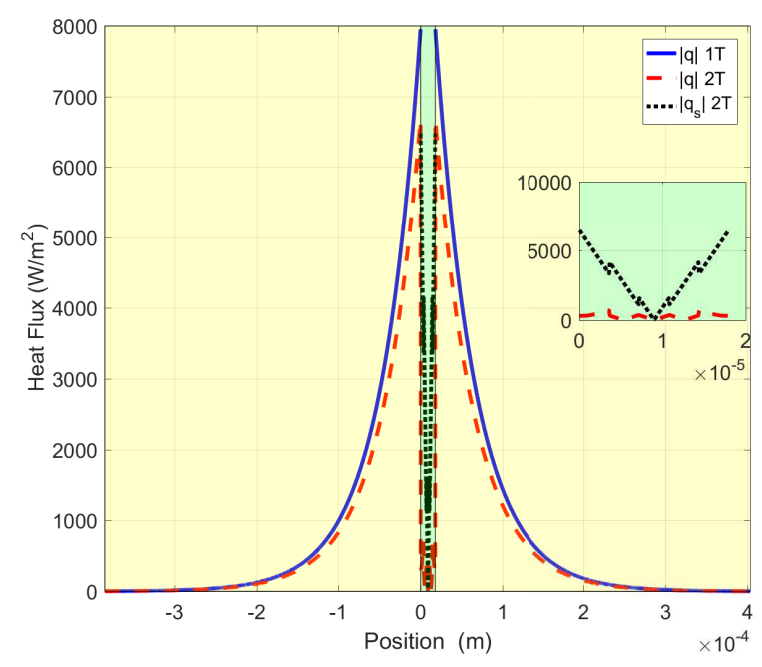

(e)

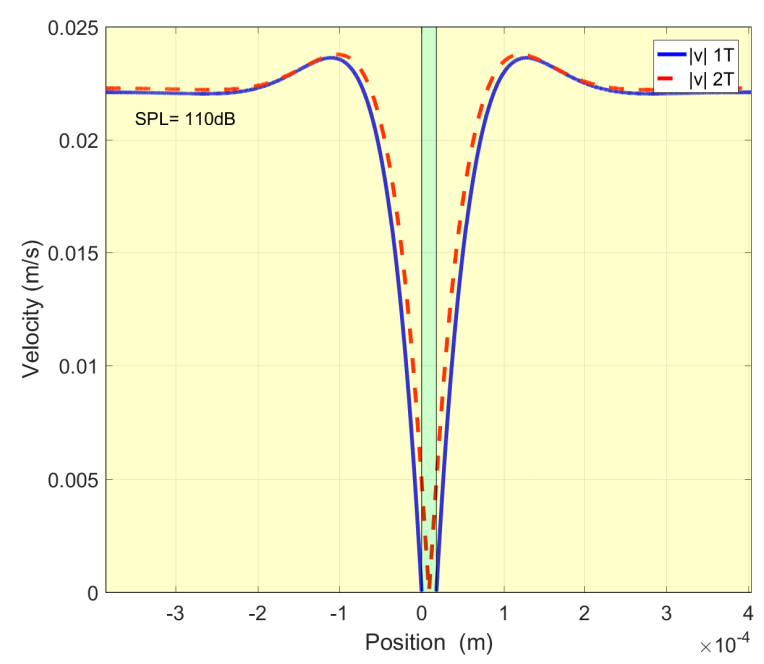

(b)

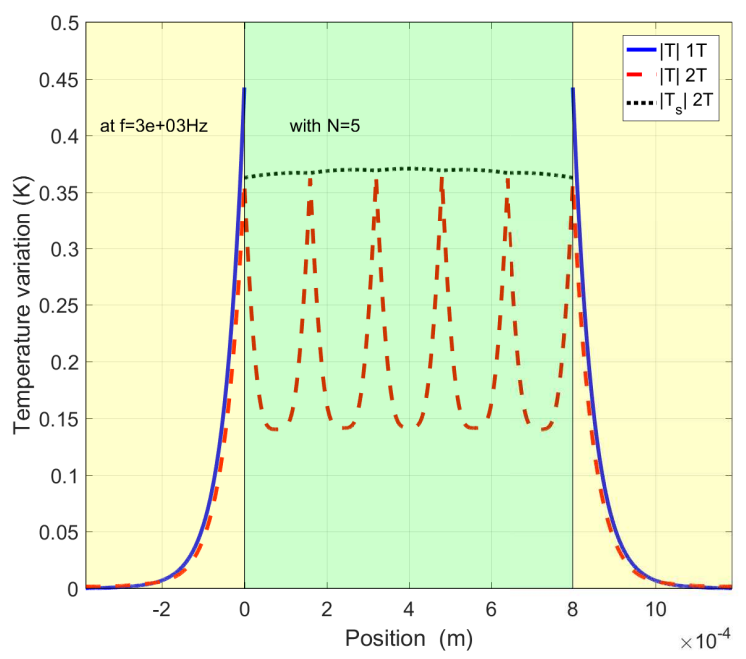

(d)

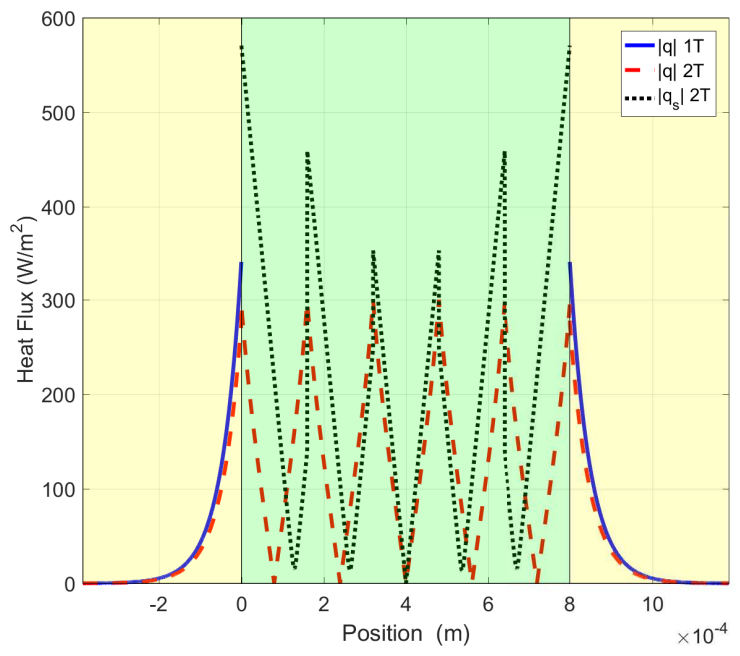

(f)

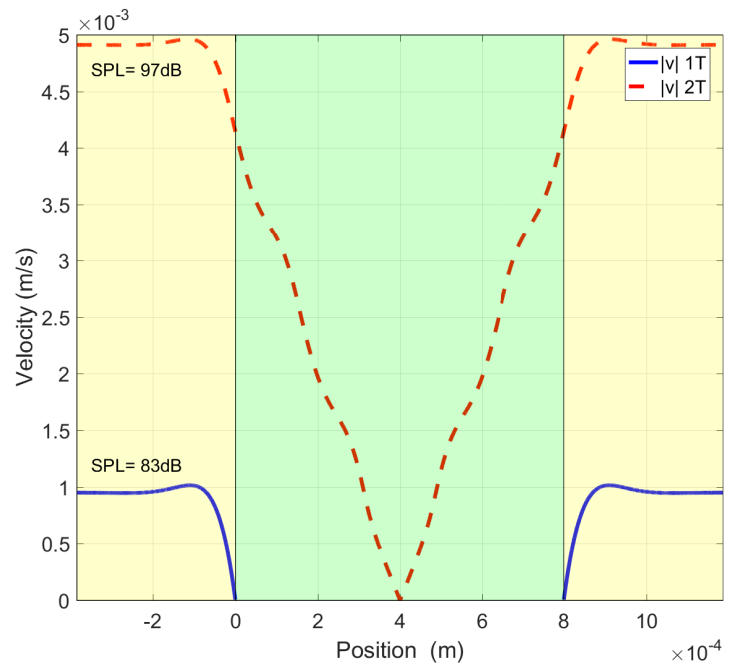

FIG. 4. Near field temperature variations (a,b), heat flux (c,d) and particle velocity (e,f) for a thin (a,c,e) and thick (b,d,f) thermophone. All parameters are plotted only in air with the $1 \mathrm{~T}$ model and in both air and solid with the $2 \mathrm{~T}$ model. All curves show the absolute value of the corresponding complex quantities. The central region (green) represents the thermophone layer with both air and solid foam and the regions on the left and on the right (yellow) represent the air layers. The insets in (a) and (c) show a zoom within the generating layer. 
(a)

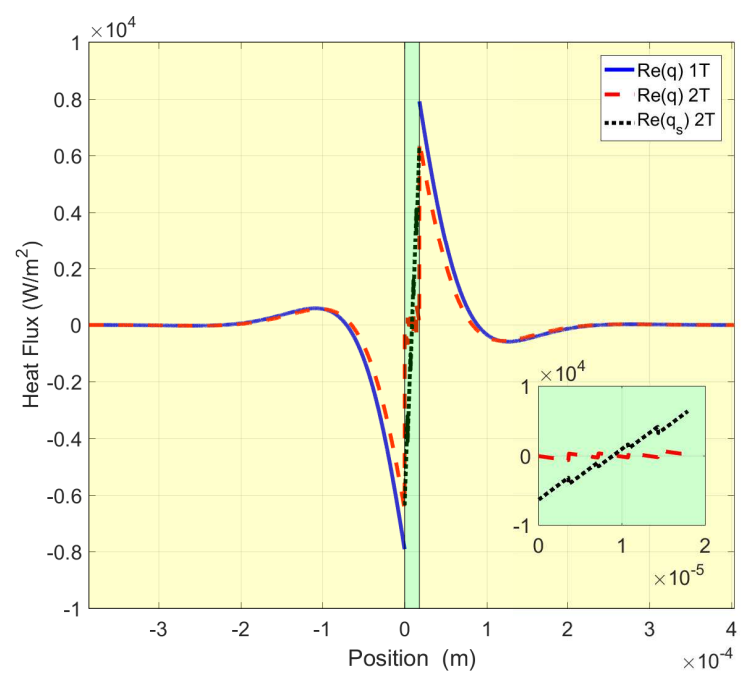

(c)

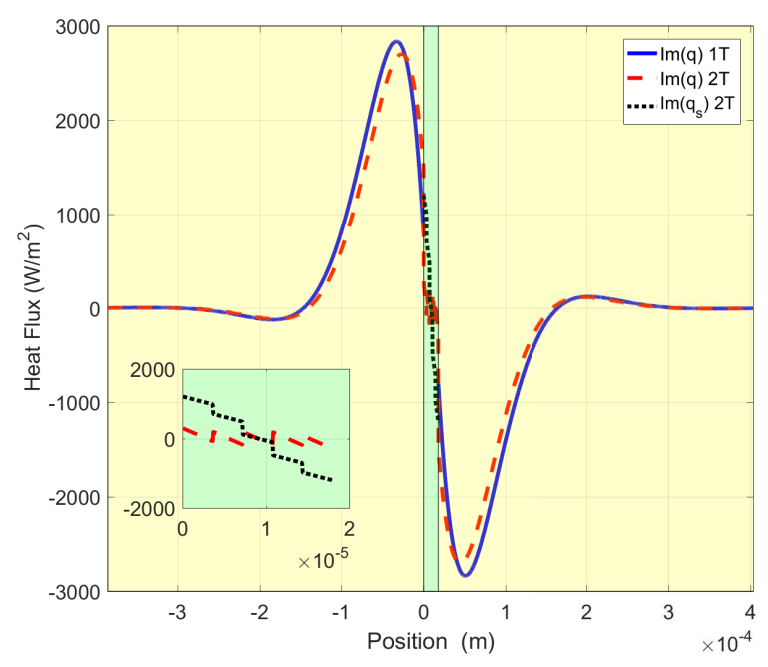

(b)

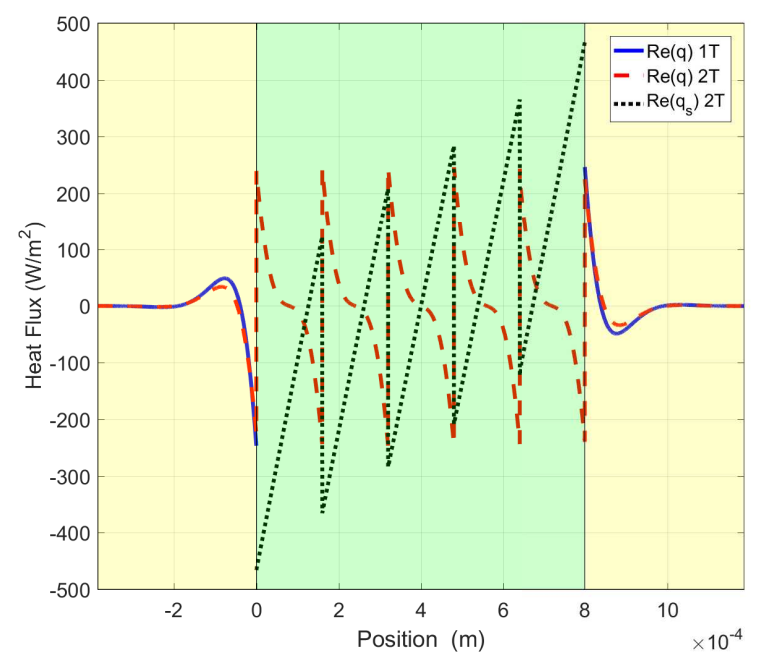

(d)

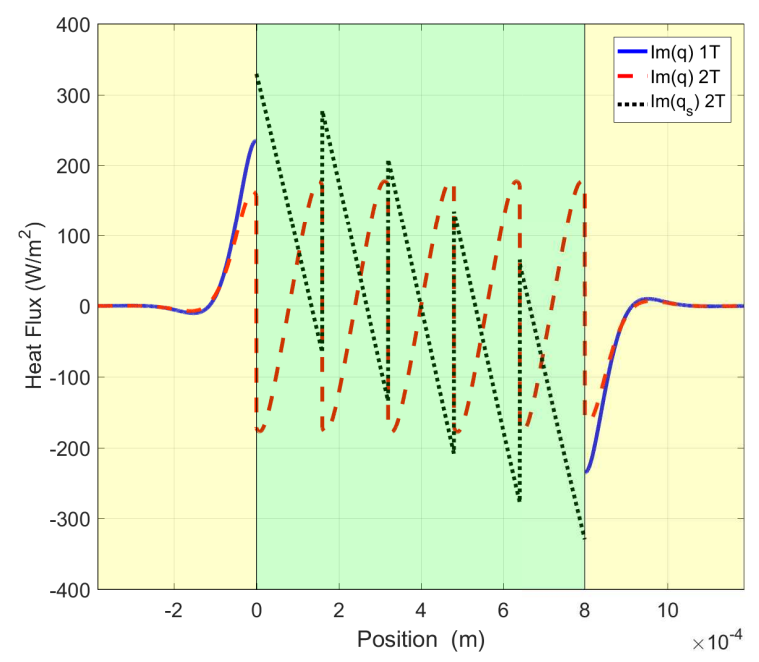

FIG. 5. Real part (a,b) and imaginary part (c,d) of the heat flux for a thin $(a, c)$ and a thick $(b, d)$ thermophone. The quantities are plotted only in air with the $1 \mathrm{~T}$ model and in both air and solid with the $2 \mathrm{~T}$ model. The central region (green) represents the thermophone layer with both air and solid foam and the regions on the left and on the right (yellow) represent the air layers. The insets in (a) and (c) show a zoom within the generating layer.

the vicinity of the thermophone and has the same length in all cases since it only depends on the frequency and the propagating medium (the higher the frequency the smaller the size of the generating layer). In the inset of Fig.4a, one can see that the temperatures inside the thin thermophone are almost constant and the branches providing the energy from the solid to the air in the $2 \mathrm{~T}$ model are not distinguishable. On the other hand, for a thick thermophone one can find in Fig. $4 \mathrm{~b}$ the energy transmission near the branches, represented by the interfaces. Finally it is seen that, in the thick thermophone, the temperature of the solid is higher at the center of the thermophone and local maxima also exist in between two branches. Importantly, these evaluations of the temperatures within the thermophone layer (pores and branches) can be performed only with the proposed $2 \mathrm{~T}$ model. These results are relevant for both analyzing the system and designing porous thermophones with specific features.

Fig.4 (panels c and d) show the heat fluxes in the considered structures. As before, the 1T model presents higher values of $q$ at the edge of the thermophone than the 2T model, but the same order of magnitude and behavior are observed in both models. The thin thermophone displays an almost continuous increase of $q$ inside the thermophone, from the centre to the external edges (2T model). However, for the thick thermophone, the heat flux transmission is seen in the air/foam structure and is characterized by a series of peaks. The sawtooth shape of the curves in Fig.4d is due to the fact that the absolute value of complex quantities is shown. To better understand the behavior of the heat fluxes in the structure, the real and imaginary parts of these quantities are represented in Fig.5, for both 
(a)

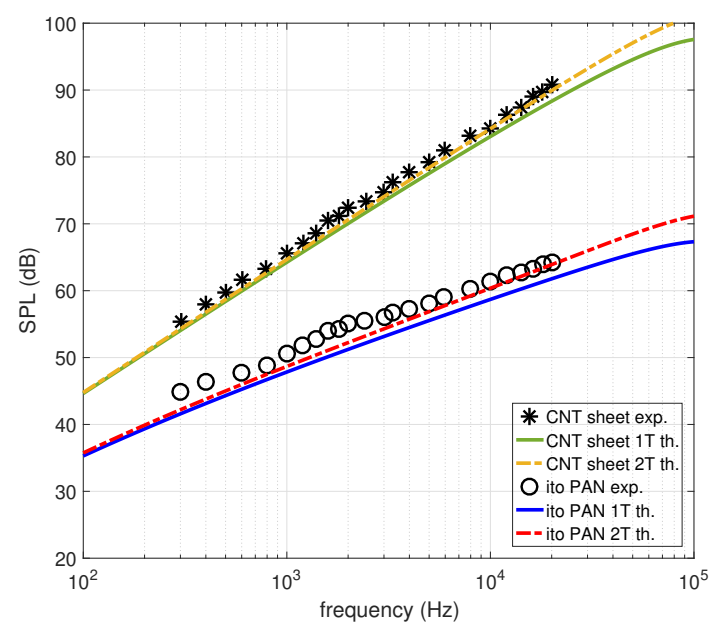

(b)

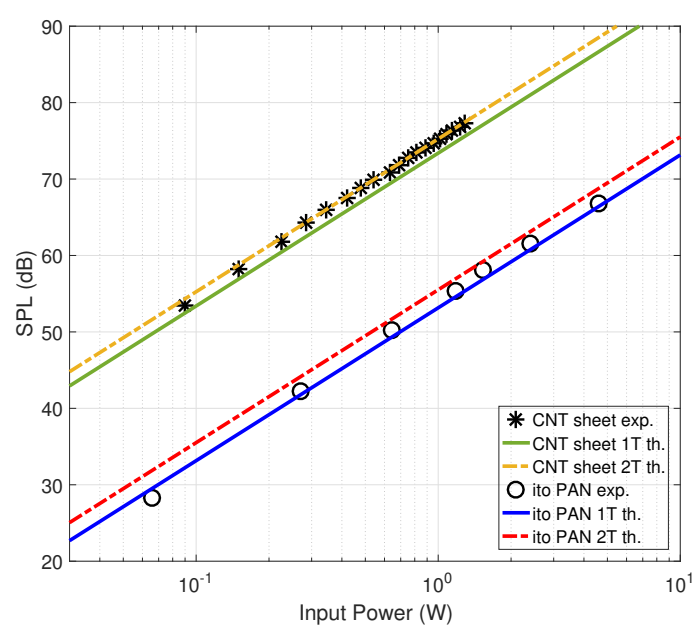

FIG. 6. Comparison of experimental ${ }^{31}$ and theoretical responses of a CNT sheet and an ITO PAN thermophone working in free field at $3 \mathrm{~cm}$ distance emission/reception. (a) Power normalized frequency response of the thinfilm thermophones. (b) SPL response of the thinfilm thermophones at $3 \mathrm{kHz}$ as a function of the input power. For the $2 \mathrm{~T}$ model $N=10$ has been adopted.

thin and thick thermophones. In the generating layer of the thin thermophone (panels a and c of Fig.5), the exchange of energy at the contact zones between air and foam can be only slightly appreciated. However, concerning the thick thermophone (panels b and d of Fig.5), one can clearly observe the jumps of the heat fluxes within the generating layer in both air and foam. These jumps represent the exchange of energy between air and foam and are described by Eqs.(16) and (17). It can be remarked that $T$ in the air is not very different from $T_{s}$ in the foam in the contact zones (see Fig.4b). Nevertheless, since the parameter $g$ assume a quite large value, the product $g\left(T_{s}-T\right)$, characteristic of the two temperature model, is always finite and positive and it can be seen (in panels b and $\mathrm{d}$ of Fig.5) as the measure of the jumps in both $q$ and $q_{s}$ curves. Moreover, it is interesting to note that the jumps in $q$ and $q_{s}$ (both real and imaginary parts) are always of the same extent but in opposite directions. It is perfectly coherent with the idea of energy exchanges, as introduced in Eqs.(16) and (17).

Fig.4 (panel e) shows that, for a thin thermophone, the particle velocity within the $1 \mathrm{~T}$ and the $2 \mathrm{~T}$ models is of the same magnitude leading to a similar radiated SPL of about $110 \mathrm{~dB}$ in the vicinity of the thermophone. This is attributed to the fact that the density of branches is high enough for the temperature and the flux to be continuous in the thermophone leading the $2 \mathrm{~T}$ model to perform similarly to the $1 \mathrm{~T}$ one. On the other hand, in Fig.4 (panel f) it is seen that the particle velocity assumes different values for the $1 \mathrm{~T}$ and the $2 \mathrm{~T}$ models, leading to different radiated SPL of about 83 and $97 \mathrm{~dB}$, respectively. This difference is attributed to the non continuity of the thermophone layer in the $2 \mathrm{~T}$ model, in opposition to the bulk solid layer in the 1T model. The improvement of the performances is clearly proportional to $N$. From the physical point of view, it means that the crucial factor to improve the performances is the total surface contact between air and foam branches within the porous generating layer. Clearly, this total surface increases with porosity but also depends on the real geometry of the microstructure (shape and connectivity of pores). The thick porous structure allows indeed to reduce the influence of the heat stored in the generating layer, thus improving the conversion of thermal energy in acoustic energy, e.g. the overall efficiency. This mechanism, properly implemented in the $2 \mathrm{~T}$ model, corresponds to the actual behavior of real porous thermophones, as proved by the following comparisons with recent experiments.

In this section it was seen that for thin thermophones the $2 \mathrm{~T}$ model performs similarly to the 1T model, but this is not the case with thicker and porous thermophones, where a sound generation difference of more than $10 \mathrm{~dB}$ is observed. This proves that the modeling of the generating layer is of primary importance and that the thermoacoustic generation is intricately linked to its geometry (size, microstructure and so on). The theoretical model has therefore to be tuned to each thermophone geometry.

\section{B. Thin film thermophones}

Fig.6 (panels a and b) shows the frequency and the power response of a Carbon Nanotube Sheet (CNT sheet) and an Indium-Tin Oxyde coated Poly(acrylonitrile) Nanofibers sheet (ITO PAN). In both cases the diffraction procedure of Section III has been applied to the theoretical models. CNT sheets are considered as the most efficient thermophones currently available because of the very low heat capacity per unit area (HCPUA) induced by their low density and low specific heat. ${ }^{31}$ For this reason, CNT sheets are considered as the reference nanostructures for thermoacoustic heaters. ${ }^{31}$ On the contrary, ITO PAN devices have a much higher density and therefore a higher HCPUA leading to a lower efficiency. This explains the different slopes seen in Fig.6 (panel a), where 
(a)

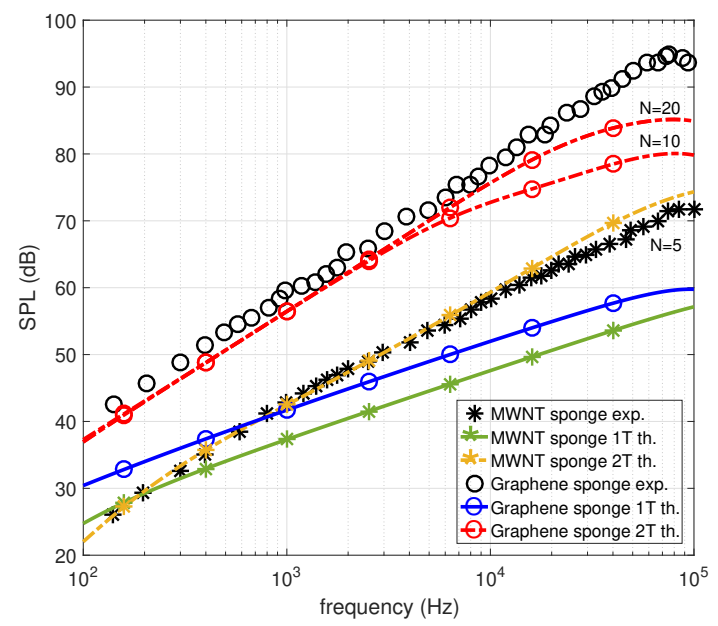

(b)

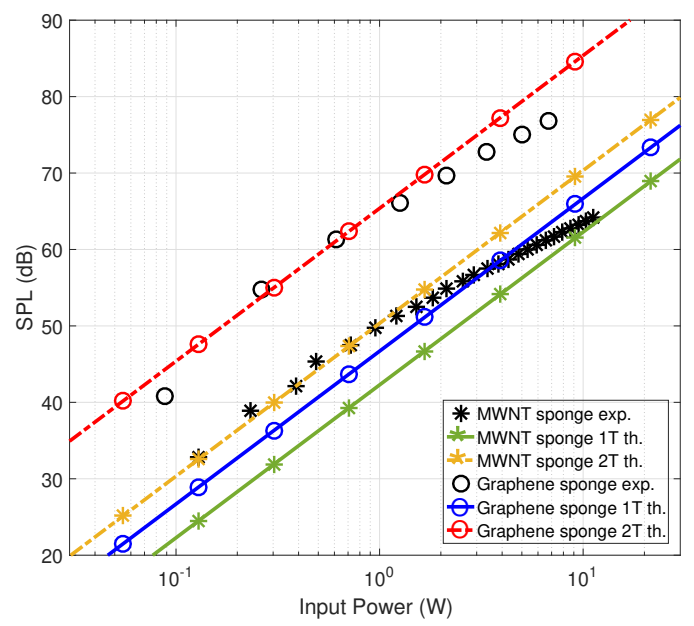

FIG. 7. Comparison of experimental ${ }^{31}$ and theoretical responses of a MWNT sponge and a graphene sponge thermophones working in free field at $3 \mathrm{~cm}$ distance emission/reception. (a) Power normalized frequency response of the foam thermophones. (b) SPL response of the foam thermophones at $3 \mathrm{kHz}$ as a function of the input power. For the graphene sponge, the theoretical curve is the same for $N=10$ and $N=20$. For the MWNT sponge $N=5$ is adopted.

the CNT sheet response has a slope proportional to $f^{1}(20$ $\mathrm{dB} / \mathrm{dec})$, whereas the ITO PAN sheet has a slope closer to $f^{0.5}(10 \mathrm{~dB} / \mathrm{dec})$ due to its higher HCPUA. ${ }^{20,31}$ This poorer efficiency is also seen in Fig.6 (panel b), where for a similar input power the ouput SPL is about 20dB higher for the CNT sheet. It is also seen that the power response slope is in both cases $20 \mathrm{~dB} / \mathrm{dec}$, meaning that the SPL output is directly proportional to the input $P_{\text {in }}$ as previously recorded in theoretical and experimental literature. ${ }^{35}$ In spite of the lower thermoacoustic performances, ITO PAN sheets are interesting materials for technological reasons. In fact, PAN polymers can be easily electrospun and coated with metals. ${ }^{31}$ The ITO coating, in particular, is deposited by radio frequency sputtering and the final film is resistant to relatively high temperatures and quite transparent. $^{31}$

A good agreement of both $1 \mathrm{~T}$ and $2 \mathrm{~T}$ models is observed with the experimental data, for frequency and power spectra. This is attributed to the fact that the thickness of the thermophone is small enough so that the $2 \mathrm{~T}$ approach does not add any significant value to the model. The thickness of the sample is still sufficiently small for the HCPUA to be accurately evaluated by a continuous sheet through a $1 \mathrm{~T}$ model. This however would stop to be true for thicker thermophones.

Finally, it can be noted that the output acoustic power for a spherical radiation on the CNT sheet can be estimated as $4 \pi r^{2} p_{\text {rms }}^{2} / \rho C_{0}=42 \times 10^{-9} \mathrm{~W}$. Here, the pressure at $1 \mathrm{kHz}$ is $p_{\text {rms }}=10^{65 / 20} \times 20 \times 10^{-6} \mathrm{~Pa}$ and $r=0.03 \mathrm{~m}$ as the measurement was done at $3 \mathrm{~cm}$ and not at the standard $1 \mathrm{~m}$ distance. The pressure being normalized at $1 \mathrm{~W}$ input power, this leads to an efficiency of about $4.2 \times 10^{-6} \%$, which is in the same range as reported in the literature. ${ }^{31}$

\section{Thick foam thermophones}

Fig.7 shows the measured responses of a MWNT sponge $(0.1 \mathrm{~mm}$ thick) and of a graphene sponge ( $0.8 \mathrm{~mm}$ thick), together with the corresponding theoretical results. In both cases the diffraction procedure of Section III has been applied. These samples have a thickness one/two orders of magnitude larger than the previous ones and have a porosity within the range $95 \%-99 \%$. The experimental frequency spectrum of the graphene sponge displays a slope proportional to $f^{1}$ $(20 \mathrm{~dB} / \mathrm{dec})$, meaning that the efficiency is preserved with respect to the thin film case. Indeed, even if the sample is rather thick, the HCPUA did not increase significantly to reduce the thermophone performances. The high frequency decrease observed is simply due to the diffraction caused by the geometry of the sample. Now, concerning the MWNT sponge experimental frequency response, one observes that for low frequencies (below $1 \mathrm{kHz}$ ) the slope is proportional to $f^{1}(20 \mathrm{~dB} / \mathrm{dec})$, and for higher frequencies (between $1 \mathrm{kHz}$ and $100 \mathrm{kHz})$ the slope becomes proportional to about $f^{0.75}(15$ $\mathrm{dB} / \mathrm{dec}$ ). Indeed, at high frequency the effect of the HCPUA increases and, therefore, the thermoacoustic generation is coherently reduced. ${ }^{20}$ Interestingly, the same effect exists also in the graphene sponge, but it is not observed here since it appears at higher frequencies because of the different physical parameters.

The important reason for introducing MWNT sponges is that MNWT networks generate an elastically compressible and flexible device. ${ }^{31}$ Moreover, the MWNT sponges fabrication is simple and low-cost. On the other hand, the use of graphene sponges allows exploiting the exceptional properties of graphene, with a very large exchange surface due to the peculiar sponge geometry. The result is a three-dimensional cross-linked sponge with isotropic physical properties. ${ }^{31}$ 
In Fig.7 (panel a), using the homogenized parameters of the thermophone shown in Table II, ${ }^{31}$ good agreement is found between the experiments and the $2 \mathrm{~T}$ theoretical model for the MWNT sponge. A good agreement is also found for low frequencies between the graphene sponge and the $2 \mathrm{~T}$ model. However, it is seen that for higher frequencies a larger number of interfaces has to be used to reproduce more accurately the experimental behavior. This is explained by the high surface density of the foam within the thermophone layer. Many interfaces need to be used to better represent the large amount of energy provided to the air for high frequencies. On the other hand, the 1T model displays a strongly different spectrum slope with respect to the experiments. This is due to the thickness of the sample. Indeed, the thermal interactions between the air and the thermophone microstructure are not integrated in the 1T model, since it assumes a thick continuous medium, thus artificially increasing the HCPUA. The sound generation inside the pores of the foam is not taken into account since the model is continuous and, therefore, more heat is assumed to be stored while it is actually dissipated in the pores air.

The power spectra of the thick samples are shown in Fig.7 (panel b), and display a different behavior with respect to the thin samples. At low input power, the SPL of the samples is proportional to $P_{i n}(20 \mathrm{~dB} / \mathrm{dec})$ but for higher input power the spectrum is approximately proportional to $P_{\text {in }}^{0.75}(15 \mathrm{~dB} / \mathrm{dec})$. This non linear behavior can be interpreted with an increase of the average static temperature inside the pores of the foam, and with a consequent efficiency reduction. ${ }^{31}$ It is seen that our model is not able to reproduce this behavior since the average temperature within the pores is fixed a priori.

\section{CONCLUSIONS}

A two temperature model for describing the thermoacoustic generation of sound by thick foam thermophones has been elaborated. To do this, the balance equations for a fluid embedded in the foam microstructure of the thermophone were used and combined with the energy balance within the solid foam material. The energetic coupling between solid foam and fluid (typically air) is controlled by a new coefficient, which describes the heat transfer between foam and air in the porous structure. For the sake of simplicity, this model is developed under the hypotheses of one-dimensional geometry and time-harmonic regime. It means that the propagation of fully coupled thermo-acoustic plane waves is investigated in the thermophone system. To model the complex microstructure, a series of interfaces are used to represent the contact zone between air and foam, where the energy transfer between the phases occurs. The resulting equations were solved by imposing the continuity of normal stress, particle velocity, temperatures and the balance of the heat flux at each interface. The calculated velocity was then used to simulate acoustic diffraction from a finite size thermophone using the classical Rayleigh's integral.

Then, the spatial distribution of the main physical variables were studied for both thick and thin thermophones. The re- sults obtained through our two temperature model were compared with the classical model composed of a solid bulk generating layer. It was proven that the output SPL may be different between these models, depending on the thickness of the thermophone. For thin thermophone, 1T and 2T model give similar results but thicker thermophones lead to significant differences in the output SPL. These differences have been explained in terms of the specific features implemented in the two models. Afterward, the frequency and power spectra were compared with experimental results published in the recent literature. ${ }^{31}$ The similar behaviors of the $1 \mathrm{~T}$ and $2 \mathrm{~T}$ models for thin thermophones were confirmed and both models were in quite good agreement with the experimental results. Thick thermophones were then investigated. While the $1 \mathrm{~T}$ model was unable to accurately reproduce the experimental results, the $2 \mathrm{~T}$ model displayed good agreement from the point of view of both frequency and power spectra. However, it could be further improved since it is unable to represent the losses due to the static temperature raise within the porous structure. It was pointed out that for complex foam like structures it is important to consider the so called homogenized parameters of the whole sample (solid plus air), which are different from the local parameters of a single foam branch.

In conclusion, the two temperatures model presented in this paper appears to be a first step in modeling thick and nano- or micro-structured thermophone systems. The most important point introduced concerns the exchange of energy at any contact surface between air and solid foam. This feature better represents the reality of such systems and is able to reproduce experimental results, which were not understood on the basis of previous models.

\section{ACKNOWLEDGMENTS}

This work was founded by Thales Underwater System and the French DGA (Direction Générale de l'Armement) and is part of a project developed in collaboration with CINTRA, UMI 3288 CNRS/NTU/THALES (Singapore).

\footnotetext{
${ }^{1}$ P. Lange, Proc. R. Soc. London. 91, 239 (1915).

${ }^{2}$ H. Arnold and I. Crandall, Phys. Rev. 10, 22 (1917).

${ }^{3}$ E. Wente, Phys. Rev. 19, 333 (1922).

${ }^{4}$ H. Shinoda, T. Nakajima, K. Ueno, and N. Koshida, Letters to Nature 400, 853 (1999).

${ }^{5}$ A. Niskanen, J. Hassel, M. Tikander, P. Maijala, L. Gronberg, and P. Helisto, Applied Physics Letters 95, 163102 (2009).

${ }^{6}$ R. Dutta, B. Albee, W. V. D. Veer, T. Harville, K. Donovan, D. Papamoschou, and R. Penner, J. Phys. Chem. C 118, 29101 (2014).

${ }^{7}$ H. Tian, D. Xie, Y. Yang, T. Ren, Y. Lin, Y. Chen, Y. Wang, C. Zhou, P. Peng, L. Wang, and L. Liu, Appl. Phys. Lett. 99, 253507 (2011).

${ }^{8}$ H. Tian, T. Ren, D. Xie, Y. Wang, C. Zhou, T. Feng, D. Fu, Y. Yang, P. Peng, L. Wang, and L. Liu, ACS Nano 5, 4878 (2011).

${ }^{9}$ W. Fei, J. Zhou, and W. Guo, small 11, 2252 (2015).

${ }^{10}$ L. Tao, H. Sun, Y. Liu, Z. Ju, Y. Yang, and TL.Ren, Appl. Phys. Lett. 111, 103104 (2017).

${ }^{11}$ A. Aliev, M. Lima, S. Fang, and R. Baughman, Nano Lett. 10, 2374 (2010).

${ }^{12}$ L. Xiao, Z. Chen, C. Feng, L. Liu, Z. Bai, Y. Wang, L. Qian, Y. Zhang, Q. Li, K. Jiang, and S. Fan, Nano Lett. 8, 4539 (2008).

${ }^{13}$ M. Daschewski, R. Boehm, J. Prager, M. Kreutzbruck, and A. Harrer, Journal of Applied Physics 114, 114903 (2013).
} 
${ }^{14}$ P. Torraca, L. Larcher, M. Bobinger, P. Pavan, B. Seeber, and P. Lugli, Journal of Applied Physics 121, 214502 (2017).

${ }^{15} \mathrm{H} . \mathrm{Hu}, \mathrm{T}$. Zhu, and J. Xu, Appl. Phys. Lett. 96, 214101 (2010).

${ }^{16}$ Y. Yin and H. Hu, AIP Conference Proceedings 1829, 020033 (2017).

${ }^{17}$ H. Hu, D. Wang, and Z. Wang, AIP Advances 4, 107114 (2014).

${ }^{18}$ C. Lim, L. Tong, and Y. Li, Journal of Sound and Vibration 332, 5451 (2013).

${ }^{19}$ L. Tong, C. Lim, S. Lai, and Y. Li, Applied Thermal Engineering 86, 135 (2015).

${ }^{20}$ P. Guiraud, S. Giordano, O. Bou-Matar, P. Pernod, and R. Lardat, J. Sound Vibr. 455, 275 (2019).

${ }^{21}$ M. Loeblein, R. Tay, S. Tsang, W. Ng, and E. Teo, Small 10, 2992 (2014).

${ }^{22}$ J. Auriault and P. Royer, Int. J. Heat Mass Transfer 36, 2613 (1993).

${ }^{23}$ M. Quintard, M. Kaviany, and S. Whitaker, Adv. Water Resour. 20, 77 (1997).

${ }^{24}$ A. Nouri-Borujerdi, A. Noghrehabadi, and D. Rees, Int. J. Heat Mass Transfer 50, 3244 (2007).

${ }^{25}$ A. d'Hueppe, M. Chandesris, D. Jamet, and B. Goyeau, Int. J. Heat Mass Transfer 55, 2510 (2012).
${ }^{26}$ S. Giordano and F. Manca, Int. J. Heat Mass Transfer 78, 189 (2014).

${ }^{27}$ L. Landau and E. Lifschitz, Fluid Mechanics, 3rd ed., Course of Theoretical Physics, Vol. 6 (Butterworths Heinemann, Oxford, 1986).

${ }^{28}$ B. Mason, S. Chang, J. Chen, S. Cronin, and A. Bushmaker, ACS Nano 9, 5372 (2015).

${ }^{29}$ F. Pavanello, F. Manca, P. Palla, and S. Giordano, Journal of Applied Physics 112, 084306 (2012).

${ }^{30}$ F. Pavanello and S. Giordano, Journal of Applied Physics 113, 154310 (2013).

${ }^{31}$ A. Aliev, N. Mayo, M. Andrade, R. Robles, S. Fang, R. Baughman, M. Zhang, Y. Chen, J. Lee, and S. Kim, ACS Nano 9, 4743 (2015).

${ }^{32}$ A. Peigney, C. Laurent, E. Flahaut, R. Bacsa, and A. Rousset, Carbon 39, 507 (2001).

${ }^{33} \mathrm{~S}$. Drieschner, M. Weber, J. Wohlketzetter, J. Vieten, E. Makrygiannis, B. M. Blaschke, V. Morandi, L. Colombo, F. Bonaccorso, and J. A. Garrido, 2D Mater. 3, 045013 (2016).

${ }^{34}$ C. Silvestri, M. Riccio, R. H. Poelma, B. Morana, S. Vollebregt, F. Santagata, A. Irace, G. Q. Zhang, and P. M. Sarro, Nanoscale 8, 8266 (2016).

${ }^{35}$ V. Vesterinen, A. Niskanen, J. Hassel, and P. Helisto, Nano Lett. 10, 5020 (2010). 